\title{
Solar flares observed simultaneously with SphinX, GOES and RHESSI
}

\author{
Tomasz Mrozek ${ }^{1,2}$, Szymon Gburek ${ }^{1}$, Marek Siarkowski ${ }^{1}$, Barbara \\ Sylwester ${ }^{1}$, Janusz Sylwester ${ }^{1}$, Anna Kępa ${ }^{1}$ and Magdalena Gryciuk ${ }^{1}$ \\ ${ }^{1}$ Solar Physics Division, Space Research Centre, Polish Academy of Sciences, ul. Kopernika 11, \\ 51-622 Wrocław, Poland \\ email: tmrozek@cbk.pan.wroc.pl \\ ${ }^{2}$ Astronomical Institute, University of Wrocław, ul. Kopernika 11, 51-622 Wrocław, Poland
}

\begin{abstract}
In February 2009, during recent deepest solar minimum, Polish Solar Photometer in $\mathrm{X}$-rays (SphinX) begun observations of the Sun in the energy range of $1.2-15 \mathrm{keV}$. SphinX was almost 100 times more sensitive than GOES X-ray Sensors. The silicon PIN diode detectors used in the experiment were carefully calibrated on the ground using Synchrotron Radiation Source BESSY II. The SphinX energy range overlaps with the Ramaty High Energy Solar Spectroscopic Imager (RHESSI) energy range. The instrument provided us with observations of hundreds of very small flares and X-ray brightenings. We have chosen a group of solar flares observed simultaneously with GOES, SphinX and RHESSI and performed spectroscopic analysis of observations wherever possible. The analysis of thermal part of the spectra showed that SphinX is a very sensitive complementary observatory for RHESSI and GOES.
\end{abstract}

Keywords. Sun: corona, Sun: flares, Sun: X-rays, gamma rays

\section{Introduction}

The first observations of solar X-ray radiation were performed more than 60 years ago (Friedman et al. 1951). Since that time we learned how to construct instruments that provide us with excellent temporal, spatial and energy resolutions. However, we rarely have occasion for observing solar flares with two or more instruments and within the overlapping energy intervals. Such common observations of the Sun with different instruments in the range of $1-20 \mathrm{keV}$ were performed several times in the past (Farnik et al. 1984, Jain et al. 2006, Gburek et al. 2008, McTiernan 2009, Väänänen et al. 2009, Kulinová et al. 2011, Sylwester et al. 2011).

In 2009 the Russian CORONAS-Photon (Kotov 2011) satellite was launched carrying a Polish spectrophotometer SphinX (Sylwester et al. 2008, Gburek et al. 2012). The energy band covered by SphinX was $1.2-15 \mathrm{keV}$ thus the excellent opportunity appeared for the comparison with low-energy end of RHESSI spectra and GOES observations. Here we present examples of common SphinX and RHESSI observations of four consecutive small solar events observed within the same active region on July $4^{\text {th }}, 2009$ around 13:55 UT. All flares were of GOES B class, providing enough photon statistics for RHESSI and without causing saturation of the SphinX D1 detector.

\section{Results and conclusions}

Performed investigation of selected flares shows that SphinX is a very sensitive complementary observatory for RHESSI and GOES. The data obtained are of good quality and are available on-line (http://156.17.94.1/sphinx_l1_catalogue/SphinX_cat_main.html). 


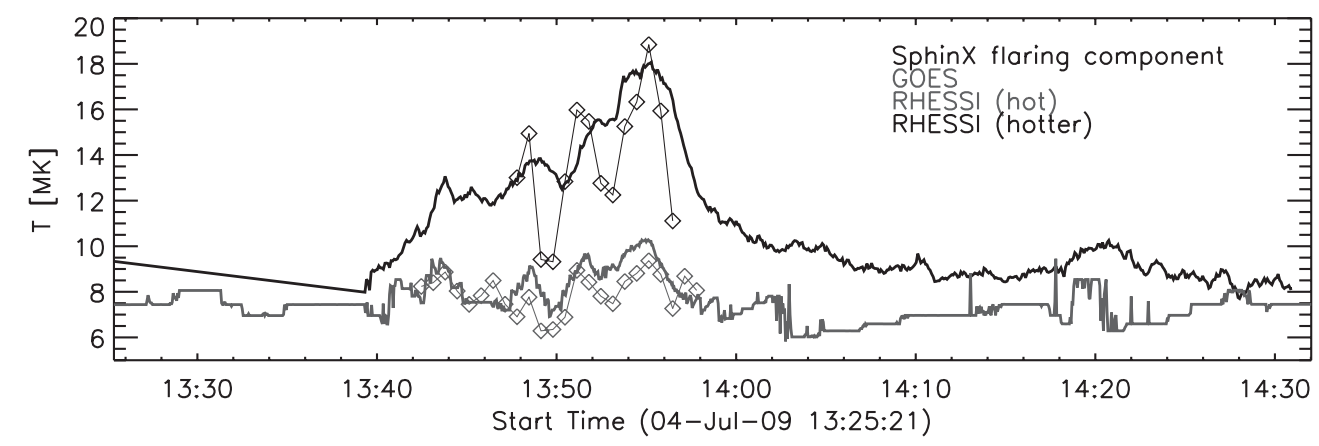

Figure 1. The example of the result of analysis for flares observed on July 4th, 2009 around 13:55 UT. Temperatures obtained from three instruments: GOES (gray curve), SphinX (black curve) and two components from RHESSI: hot (thin black curve + diamonds) and hotter (gray curve + diamonds).

The temperature and emission measure for SphinX and RHESSI were obtained from spectral fitting to observed data. Emitting volumes were estimated from RHESSI images. These parameters were used for estimation of thermal energy and thermodynamical measure: $\eta=3 k T \sqrt{E M}$ (Sylwester et al. 1995). GOES temperatures were obtained with a standard filter ratio technique. The results of our analysis may be summarized as follow:

- The SphinX and RHESSI spectral responses nominally overlap in the range $3-$ $15 \mathrm{keV}$. In practice SphinX did not measure solar signals above $7-8 \mathrm{keV}$. Therefore the range of comparison performed was limited to energies $3-6 \mathrm{keV}$.

- We compared temperatures from SphinX flaring component, GOES and two thermal components obtained from fit to RHESSI spectra. RHESSI hot component behaviour follows GOES and RHESSI hotter component agrees well with SphinX flaring component. See the consistent pattern in Figure 1.

- Derived values and variability pattern of the thermodynamic measure agree very well for SphinX, GOES and RHESSI instruments.

We acknowledge financial support from the Polish National Science Centre grant 2011/01/M/ST9/06096 and from the European Commission Seventh Framework Programme under the grant agreement No. 284461 (eHEROES project)

\section{References}

Farnik, F., Valnicek, B., Sylwester, B., Sylwester, J., \& Jakimiec, J. 1984, Bulletin of the Astronomical Institutes of Czechoslovakia, 35, 158

Friedman, H., Lichtman, S. W., \& Byram, E. T. 1951, Physical Review, 83, 1025

Gburek, S., Mrozek, T., Siarkowski, M., \& Sylwester, J. 2008, Advances in Space Research, 42,822

Gburek, S., Sylwester, J., Kowalinski, M., et al. 2012, Solar Phys., 283, 631

Jain, R., Pradhan, A. K., Joshi, V., Shah, K. J., et al. 2006, Sol. Phys., 239, 217

Kotov, Y. D. 2011, Solar System Research, 45, 93

Kulinová, A., Kašparová, J., Dzifčáková, E., et al. 2011, A\&̛A, 533, A81

McTiernan, J. M. 2009, ApJ, 697, 94

Sylwester, J., Garcia, H. A., \& Sylwester, B. 1995, A\&A, 293, 577

Sylwester, J., Kuzin, S., Kotov, Y. D., Farnik, F., \& Reale, F. 2008, Journal of Astrophysics and Astronomy, 29, 339

Sylwester, B., Sylwester, J., Siarkowski, M., Engell, A. J., \& Kuzin, S. V. 2011, Central European Astrophysical Bulletin, 35, 171

Väänänen, M., Alha, L., \& Huovelin, J. 2009, Sol. Phys., 260, 479 